Journal of Case Reports 2018;8(2):102-104

\title{
Pulmonary Embolism in Young Male
}

\author{
Chiranjeevi Chintala, Gebran Manzoor Monga, Dheeraj Nair \\ Department of Emergency Medicine, Max Superspeciality Hospital, Vaishali, Ghaziabad, Uttar Pradesh, India.
}

\begin{abstract}
Corresponding Author:
Dr. Gebran Manzoor Monga

Email: jibranmonga@gmail.com

This is an Open Access article distributed under the terms of the Creative Commons Attribution License (creativecommons.org/ licenses/by/3.0).

Received : January 26, 2018

Accepted : March 20, 2018

Published : April 20,2018
\end{abstract}

\begin{abstract}
Background: Pulmonary embolism remains a disease which needs high clinical suspicion to prevent mortality and morbidity. More so in young healthy individuals, suspicion is very low as compared to old age individuals with multiple co-morbid conditions. Pulmonary embolism carries high mortality if not suspected and treatment initiated as early as possible. Case Report: This is a case report of young male who presented with acute onset of breathlessness and later diagnosed as a case of pulmonary thromboembolism. Conclusion: Pulmonary embolism should be kept a possibility in a young adult presenting with acute onset of dyspnea.
\end{abstract}

Keywords: Comorbidity, Dyspnea, Male, Humans, Pulmonary Embolism, Thromboembolism.

\section{Introduction}

Acute pulmonary embolism is a component of venous thromboembolism (VTE), which may prove fatal if not suspected and subsequently treated. Incidence of pulmonary embolism is around $0.5-1$ case per 1000 cases [1]. This has been estimated that $70 \%$ of proven post-mortem cases of pulmonary embolism are not even suspected during the course of treatment [2]. On the contrary, only $25-30 \%$ of suspected cases turned out to be cases of pulmonary embolism in post-mortem studies [3]. Young patients are more likely to be mismanaged, as suspicion in healthier young patient is very low, and inspite of various diagnostic modalities high clinical suspicion remains the key for diagnosis.

\section{Case Report}

A 30-year-old young male patient, no known comorbidity, presented to emergency department with history of cough and breathlessness on exertion for 2 days. Initial examination showed respiratory rate: $28 / \mathrm{min}$, pulse: $120 / \mathrm{min}$, blood pressure: $130 / 80 \mathrm{~mm}$ of $\mathrm{Hg}, \mathrm{SpO}_{2}: 88 \%$ on room air, temperature: $98.4^{\circ} \mathrm{F}$, and scattered wheeze on chest examination. No other significant finding was noted. CT thorax showed peripheral patchy areas of consolidation/atelectasis like changes in right middle and lower lobes and left lower lobe.

Patient was admitted and shifted to intensive care unit. He was managed with broad spectrum antibiotics, antipyretics, fluids, oxygen and supportive care. His symptoms started to worsen; he had tachypnea, persistent hypoxemia. 2D echocardiography showed mild mitral and tricuspid regurgitation with ejection fraction 55\%, right ventricular systolic pressure (RVSP) 55 $\mathrm{mmHg}$. His bilateral lower limb venous Doppler was done which did not show any evidence of deep venous thrombosis. CT pulmonary angiography was done which showed moderate pulmonary thromboembolism with evidence of hypodense thrombus seen at ramification of bilateral pulmonary arteries (right $>$ left) extending in to segmental branches [Fig.1-3]. Patient was started on low molecular weight heparin. Despite four days of low molecular weight heparin therapy patient was still having high oxygen requirement and persistent tachypnea along with hemoptysis and hematuria. His repeat 2D echocardiography showed RVSP $65 \mathrm{~mm}$ of $\mathrm{Hg}$. Now intra-arterial thrombolysis was 

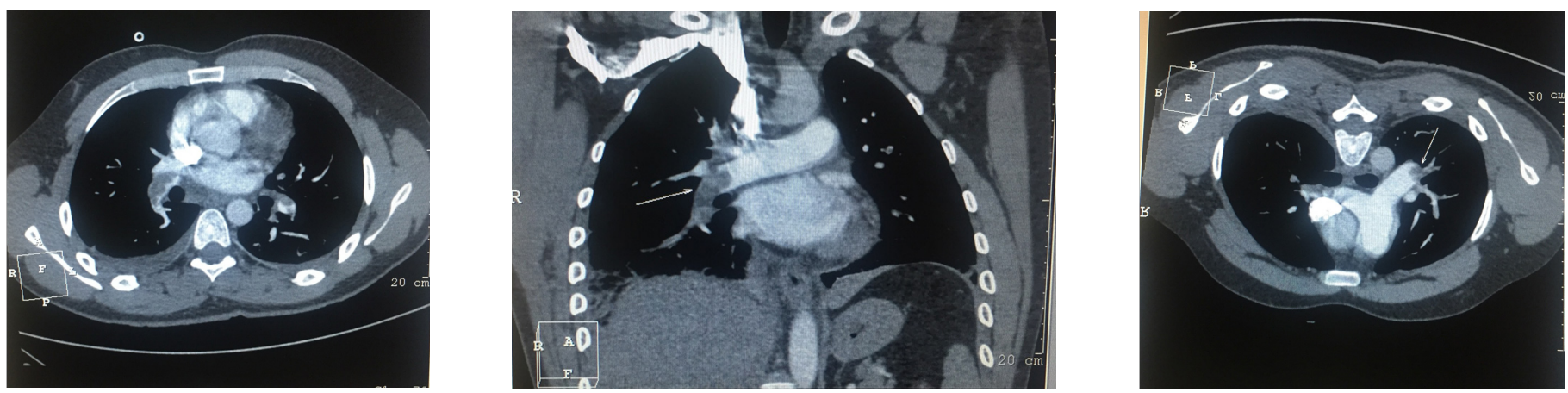

Fig.1-3: CT angio showing segmental pulmonary embolism.

done with reteplase. Patient symptoms started to improve, repeat 2D echocardiography showed RVSP of $45 \mathrm{~mm} \mathrm{Hg}$. Subsequently, patient was discharged on oral anti-coagulants.

\section{Discussion}

Pulmonary embolism is common and potentially lethal condition. Most patients who succumb to pulmonary embolism do so within the first few hours of the event. Despite diagnostic advances, delays in pulmonary embolism diagnosis are common and represent an important issue [6]. As a cause of sudden death, massive pulmonary embolism is second only to sudden cardiac death.

The risk of blood clots is increased by cancer, prolonged bed rest, smoking, stroke, certain genetic conditions, estrogen-based medication, pregnancy, obesity, and after some types of surgery [4]. About $90 \%$ of emboli are from proximal leg DVTs or pelvic vein thromboses. Clinically apparent DVT is present in only $11 \%$ of confirmed cases of pulmonary embolism. The classic presentation of PE is the abrupt onset of chest pain, breathing difficulty and hypoxia but some patients may have no obvious symptoms at presentation. The diagnosis of pulmonary embolism should be suspected in patients with respiratory symptoms unexplained by an alternative diagnosis. Evidence-based literature supports the practice of determining the clinical pre-test probability of pulmonary embolism before proceeding with diagnostic testing [4]. The three validated systems include the Modified Wells Scoring System, the Revised Geneva Scoring System, and the Pulmonary Embolism Rule out Criteria (PERC) [5-7]. Low probability PE can be ruled out with D-dimer testing [11]. CTPA is the gold standard for diagnosing pulmonary embolism [12]. When a pulmonary embolism is identified, it is characterized as acute or chronic. In terms of pathologic diagnosis, acute embolus is situated centrally within the vascular lumen and occludes a vessel (vessel cutoff sign) [12]. Chronic embolus is eccentric and contiguous with the vessel wall, reduces the arterial diameter by more than $50 \%$, evidence of recanalization within the thrombus is present, and an arterial web is present.

The variability of presentation sets the patient and clinician up for potentially missing the diagnosis. Classic presentation of abrupt onset of pleuritic chest pain, shortness of breath, and hypoxia is rarely seen. Studies of patients who died unexpectedly of pulmonary embolism revealed that patients had complained of nagging symptoms, often for weeks, before dying. Forty percent of these patients had been seen by a physician in the weeks prior to their death

Acute pulmonary embolism in young adults is not very uncommon. A young adult presenting with acute onset of dyspnea, pulmonary embolism should be kept a possibility, as most of the time $\mathrm{PE}$ is not considered in differential diagnosis while evaluating such patients in emergency. Most of 
young patients reported in literature are young females, either pregnant or on oral contraceptives. Pulmonary embolism requires high clinical suspicion, based on validated scores and requires further multi-modality investigation to confirm or rule out disease.

\section{Conclusion}

Unlike infectious diseases, cardiac disorder or blood disorder, no specific symptoms, signs or investigations reflect pulmonary embolism immediately. Hence young male presenting with acute onset of dyspnea should be evaluated for common cause of dyspnea but pulmonary embolism should be kept in mind, so as not to miss this fatal disease.

Contributors: CC: manuscript writing, patient management; GMM: manuscript editing, patient management; DN: critical inputs into the manuscript. GMM will act as guarantor. All authors approved the final version of this manuscript. Funding: None; Competing interests: None stated.

\section{References}

1. Heit JA. The epidemiology of venous thromboembolism in the community. Arteroscler Thromb Vasc Biol. 2008;28:370-372.

2. Goldhaber SZ, Hennekens CH, Evans DA, Newton EC, Goldleski JJ. Factors associated with correct antemortem diagnosis of major pulmonary embolism. Am J Med. 1982;73:822-826.

3. Rubeinstein I, Murray D, Hoffstein V. Fatal pulmonary emboli in hospitalised patients: An autopsy study. Arch Intern Med. 1988;148:1425-1426.

4. Anderson FA Jr, Spencer FA. Risk factors for venous thromboembolism. Circulation 2003;107:9-16.
5. Wells PS, Ginsberg JS, Anderson DR, Kearon C, Gent $\mathrm{M}$, Turpie AG, et al. Use of clinical model for safe management of patients with suspected pulmonary embolism. Ann Intern Med. 1998;129:997-1005.

6. Elliot CG, Goldhaber SZ, Visani L, De Rosa M. Chest radiography in acute pulmonary embolism. Results from the International Cooperative Pulmonary Embolism Registry. Chest. 2000;118:33-38.

7. Ferrari E, Imbert A, Chevalier T, Mihoubi A, Morand P, Baudouy M. The ECG in pulmonary embolism. Predictive value of negative $\mathrm{T}$ waves in precordial leads - 80 case reports. Chest. 1997;111:537-543.

8. Wells PS, Anderson DR, Rodger M, Steill I, Dreyer JF, Barnes D, et al. Excluding pulmonary embolism at the bedside without diagnostic imaging: Management of patients with suspected pulmonary embolism presenting to the emergency department by using a simple clinical model and d-dimer. Ann Intern Med. 2001;135:98-107.

9. Stein PD, Fowler SD, Goodman LR, Gottschalk A, Hales A, Hull RD, et al. Multi detector computed tomography for acute pulmonary embolism. N Engl J Med. 2006;354:2317-2327.

10. Kurzyna M, Torbicki A, Pruszczyk P, Burakowska B, Fijałkowska A, Kober J, et al. Disturbed right ventricular ejection pattern as new Doppler echocardiographic sign of acute pulmonary embolism. Am J Cardiol. 2002;90:507-511.

11. Cossete B, Pelletier ME, Carrier N, Turgeon M, Leclair $\mathrm{C}$, Charron $\mathrm{P}$, et al. Evaluation of bleeding risk in patients exposed to therapeutic unfractioned or low molecular weight heparin: A cohort study in context of a quality improvement initiative. Ann Pharmacother. 2010;44:994-1002.

12. Dalla-Volta S, Palla A, Santolicandro A, Giutini C, Pengo $\mathrm{V}$, Visioli O, et al. PAIMS 2: Alteplase combined with heparin verses heparin in treatment of acute pulmonary embolism. Plasminogen activator Italian multicentre study 2. J Am Coll Cardiol. 1992;20:520-526. 